\title{
Issues and Implications of the Thermal Control System on Responsive Space Missions
}

\author{
Andrew D. Williams \\ Air Force Research Laboratory, Space Vehicles Directorate \\ 3550 Aberdeen Ave., Kirtland AFB, NM 87117 \\ Dr. Scott E. Palo \\ University of Colorado, Department of Aerospace Engineering Sciences \\ Boulder, CO 80309
}

\begin{abstract}
One aspect that poses a significant hurdle to achieving the goals of Operationally Responsive Space (ORS) is the thermal control system (TCS). Traditionally the TCS must be vigorously designed, analyzed, tested, and optimized from the ground up for every satellite mission. This "reinvention of the wheel" is costly and time intensive. Current design cycles require years. Next generation satellite thermal management must be robust, modular, and scalable in order to cover a wide range of applications, orbits, and mission requirements. To provide a better understanding of the issues and implications of the TCS and to help bound the problem for the development of robust and modular thermal designs, a preliminary analysis was conducted to determine the upper and lower design bounds for a small responsive satellite. In addition, the range of external heat loads for small satellites in low earth orbit were evaluated. From this analysis, the worst hot and cold cases conditions were identified. Using these two cases, various design parameters were evaluated, three different design approaches were compared, and the feasibility of a one-size-fits-all approach was assessed.
\end{abstract}

\section{INTRODUCTION}

The 2001 Space Commission Report stated that "the United States (U.S.) is more dependent on space than any other nation"1. This is especially true for military applications where space is used for surveillance, communication, navigation, meteorology, theatre support, and force application. The U.S.'s use of existing space capabilities provides its forces an asymmetric edge during battle. It is also a capability that potential adversaries must plan to defend against or attack. As more nations gain access to space, the threat to U.S. assets will increase, and the ability to maintain its dominance will become more difficult. It may be impossible for the U.S. to maintain its space dominance if it continues to focus its efforts on large, multibillion dollar spacecraft that take years to design and field.

Historically, large space assets have been considered strategic in nature because they take years to design, assemble, test, and deploy. A typical large satellite takes between three and ten years to design and field. In addition, the total mission cost ranges from hundreds of millions to billions of dollars. Compounding the problems are the significant cost and schedule overruns experienced by most programs. Prime examples are the Advanced Extremely High Frequency (AEHF) military communication program and the Space-Based Infrared Systems (SBIRS) early warning satellite program. By their inherent nature, large complex systems are expensive and time intensive.
There has been a growing move in the aerospace industry and a growing need in the Department of Defense (DOD) to make space more responsive and cost effective. Instead of taking years to design and deploy a new satellite, the goal is weeks or even days. The DOD is actively pursuing the capability to make space operationally responsive. The goal is to extend the advantages space affords from the strategic planner to the battlefield commanders. The ability to launch a new space asset within days or hours of a battlefield commander's request will maintain the asymmetric advantage in future conflicts. Space provides the ultimate high ground, and Operationally Responsive Space (ORS) brings this advantage directly to the battlefield commander.

To meet this challenge, the methodologies used to design, manufacture, test, launch, and deploy satellites must radically change. For space to become operationally responsive, satellites must be easily manufactured, assembled, tested, and prepared for launch in a military depot style environment. Designs will have to be simple and robust so that Airmen play a central role and rather than Ph.D.-level scientists. Large geosynchronous satellites will continue to play an important role in space activities, but to achieve the goals of responsive space, components and systems will have to be standardized and simple, which translates to an increasing usage of small satellites. 
One of the most challenging aspects of this problem is the satellite's Thermal Control System (TCS). Traditionally, the TCS is vigorously designed, analyzed, and optimized for every satellite mission. This "reinvention of the wheel" is costly and time intensive. The next generation satellite TCS must be robust, modular, and scalable in order to meet the needs of a wide range of missions, payloads, and thermal requirements.

\section{TRADITIONAL THERMAL CONTROL SYSTEM DESIGN}

The traditional approach to satellite design is a customized and highly optimized satellite bus. The primary design driver is to minimize mass but often at the expense of time and money. This design driver is maintained throughout the design of the entire spacecraft. A secondary design driver is system reliability. Since spacecraft are expensive, complex, and nearly impossible to repair once on orbit, system reliability is also important. As a result of these two constraints, every aspect of the system's design must be carefully considered, analyzed, and tested. The result is a long and arduous design process.

The primary responsibility of the TCS is to maintain all components within their operating temperature limits throughout all mission phases. Like most other satellite systems, the requirements and constraints placed on the system are dictated by the other satellite subsystems. However, unlike most other subsystems, the TCS is a completely distributed system that is intimately interconnected with the other satellite systems. As a result, a higher level of fidelity about the overall system is required before design and analysis can commence.

The first step in the thermal design process is to determine the component temperature limits, the internal power dissipation, and the worst case environmental heat inputs. Using these inputs, the basic energy balance of the satellite is determined. Next, a simplified thermal model is developed and different TCS concepts are evaluated. At this point, the process becomes iterative. For each successive analysis, the fidelity of the thermal model is increased, and the TCS design is refined. As the design of the satellite and the components change, the thermal model must be updated. Each change must be analyzed for its effect on the TCS and the overall design of the spacecraft. Figure 1 illustrates the various inputs and the overall design process for the thermal control system. Once the detailed design and thermal model are completed, they must be validated in a thermal balance test. Using the results from the thermal balance test, the model is validated and the design is finalized. The final proof test for the system is the thermal vacuum test. The result is a process that takes years to complete and will not meet the goals of responsive space.

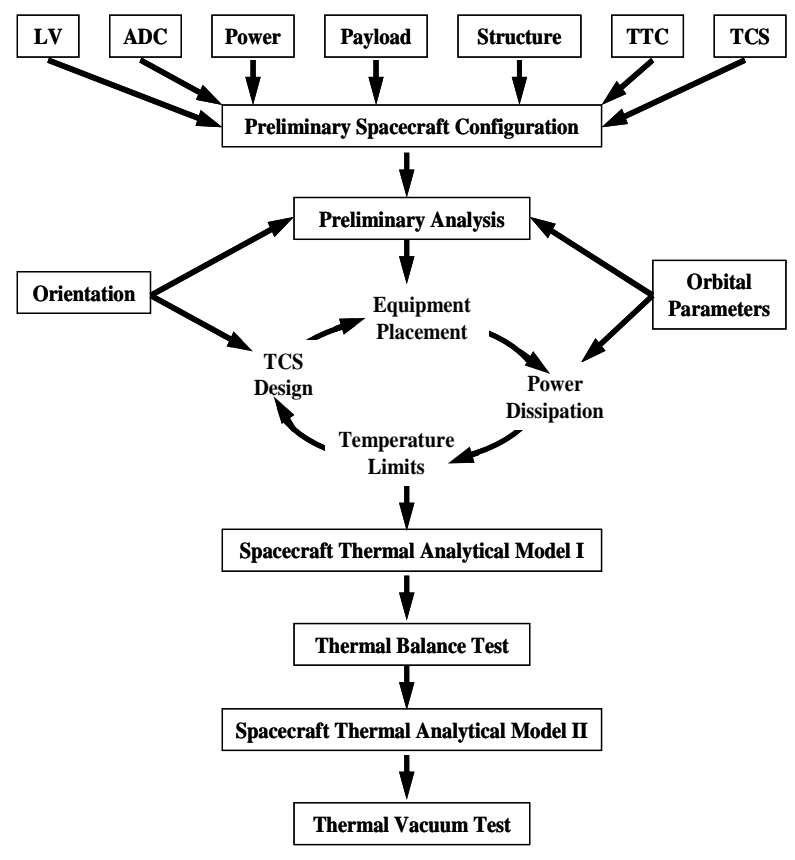

Figure 1: Overall Thermal Design Process

\section{THERMAL CONTROL SYSTEM DESIGN FOR RESPONSIVE SPACE}

For ORS mass and reliability are important design drivers, but they are not necessarily the primary drivers. The primary drivers for ORS are time and cost. The ultimate goal is a low cost system that can be launched within six days of call up from the battle field, hence the term the "six day satellite." In order to accomplish this goal, time and cost will have to be traded with mass, reliability, and other design variables. Traditional design practices will still have their place, but for operationally responsive space to be feasible, a new design paradigm is required. To meet the goals of ORS, the satellite must be modular and adaptable to different missions, changing threats, and emerging technologies. This poses a great challenge to many systems, especially the thermal control system.

Previous attempts to reduce the cost and time for bus development have focused on standardizing the bus. The disadvantage with most standardized bus development programs is that the bus eventually becomes obsolete and must be completely redesigned as new technologies are developed. One of the goals of 
the ORS program is the development of technologies that provide robust and flexible bus designs. For example, self-organizing network approaches that leverage commercial approaches are being developed for ORS avionics networks ${ }^{2}$. The concept is similar to PC based plug-and-play USB connectivity and is being investigated for the command and data handling system $^{3}$. Plug-and-play addresses the software and electrical interfaces, but other efforts are needed to address the mechanical and thermal interfaces

To achieve the goals of ORS, the satellite or the subsystem components will have to be on hand for rapid integration and launch; however, their state of pre-integration is still open for debate. There are three primary options. The first is the more flexible option in which the components are on hand so that the satellite can be quickly assembled to meet the needs of the mission. The second and faster option would be to have the satellite preassembled so that it is ready for integration to the launch vehicle. The final option is a combination of the two where the modules are preassembled and then integrated into the satellite structure based on mission needs. Because this option provides both flexibility and speed to some degree, it was used as the integration strategy for this study.

This philosophy is not new and has analogies in the computer and automotive industry. A supplier, such as GM, has a standard model that will meet the needs of the majority of the market. For those users that need additional features, such as an automatic transmission or anti-lock brakes, the appropriate upgrades are made to the standard model before the unit is shipped to the customer. For the user that requires a top of the line system, often times a custom built system provides the only economic solution. Under this philosophy, respon-sive satellites would operate in a similar fashion. A base model would be available that meets the majority of mission needs. For missions or payloads that require additional capability than provided by the baseline system, the appropriate upgrades would be made.

\section{SUBSYSTEM COMPONENTS}

Regardless of the design philosophy, a certain level of fidelity of the bus design is needed before the basic requirements for the thermal control system can be designed. Because of launch vehicle limitations, ORS missions will likely be relegated to $<450 \mathrm{~kg}$ class satellites. Using this basic assumption, the capabilities that a small satellite bus can provide can be determined. To evaluate the internal heat load that the TCS must be able to accommodate, a bus sizing exercise was conducted.

The purpose of the exercise was not to specify exact components for the bus but to identify the design space for the system based on current and near term technologies. For each subsystem, two levels of capability were identified. Similar to the Dell analogy, subsystem components were sized to provide a baseline capability and an upgraded one. From these components, the mass, volume, and power of the subsystem were estimated. The results were an upper and lower bounds for the design of the TCS and are only summarized here. A more detailed analysis can be found in Reference 4.

\section{Low Capability Bus}

The low capability bus (LCB) represents a minimum level of capability that is required for small satellite missions. It is important to note that these system requirements do not represent any particular mission or system. Instead, they are a first order approximation based on general mission needs and were used to begin subsystem design and analysis. The capability for each subsystem is summarized below.

Attitude Determination and Control (ADC)

- Attitude knowledge of $0.1^{\circ}-1^{\circ}$

- Pointing accuracy of $1^{\circ}-5^{\circ}$

- Slew rate of $0.05-0.1 \%$

Telemetry, Tracking, and Command (TTC)

- S-band system

- Data rate of $1 \mathrm{Mbps}$

Navigation and Guidance (NG)

- 12 channel GPS receiver

Command and Data Handling (CDH)

- $\quad$ Space Plug-and-play Avionics-USB (SPA-U) based system

- Legacy system compatibility

- Power management for USB based components

Power Management (PM)

- $500 \mathrm{~W}$ system

- Triple junction deployed solar array

- Lithium-ion batteries

- Peak power tracking (PPT)

Structure

- Aluminum honeycomb 
SSC06-3-1

\section{Propulsion}

- No onboard propulsion system

There are a few important points to note. First, the control scheme for the ADC system is based on a momentum bias system with magnetic torque rods providing additional control. Second, a PPT control system is used for power regulation. The advantage of a PPT system is that it acts like a low impedance power supply making design integration a simple task. Finally, because of the short mission life, an onboard propulsion system was not included in the system sizing. It is assumed that the orbit altitude will be high enough to meet mission requirements without additional station keeping.

Using these requirements, components were selected for each subsystem. The resulting mass, power, and volume requirements are summarized on Table 1 below.

Table 1: Low Capability Bus

\begin{tabular}{|l|c|c|c|}
\hline Subsystem & $\begin{array}{c}\text { Mass } \\
{[\mathrm{kg}]}\end{array}$ & $\begin{array}{c}\text { Power } \\
{[\mathrm{W}]}\end{array}$ & $\begin{array}{c}\text { Size } \\
{[\mathrm{cm}]}\end{array}$ \\
\hline ADC & 10.3 & 18.5 & $30 \times 24 \times 12$ \\
\hline TTC & 2.8 & 7.4 & $9.8 \times 9.6 \times 7.2$ \\
\hline NG & 0.02 & 0.8 & $7.0 \times 4.5 \times 1.0$ \\
\hline CDH & 15.2 & 50 & $34 \times 25 \times 20$ \\
\hline PM & 18.3 & 70.3 & $25 \times 23 \times 21$ \\
\hline Structure & 21.5 & n/a & $27 \times 40.5 \times 71$ \\
\hline Propulsion & 0 & 0 & $0 \times 0 \times 0$ \\
\hline \multirow{2}{*}{} & $\mathbf{6 8 . 1}$ & $\mathbf{1 4 7 . 0}$ & $\mathbf{2 7 \times 4 0 . 5 \times 7 1}$ \\
\cline { 2 - 4 } & \multicolumn{2}{l}{}
\end{tabular}

High Capability Bus

Opposed to the LCB, the high capability bus (HCB) does not represent the maximum capability that is required for small satellite missions. It is merely a more capable bus that is more representative of an $\sim 80 \%$ design solution. For ORS, the goal is not a $100 \%$ design solution for all scenarios. The capability for each subsystem is summarized below.

Attitude Determination and Control (ADC)

- Attitude knowledge of $0.02^{\circ}-0.1^{\circ}$

- Pointing accuracy of $0.05^{\circ}-1^{\circ}$

- Slew rate of $0.1-0.3 \%$

Telemetry, Tracking, and Command (TTC)

- $\quad$ S-band system for housing keeping

- Ku-band CDL system

- Data rate of $274 \mathrm{Mbps}$
- 12 channel GPS receiver

Command and Data Handling $(\mathrm{CDH})$

- $\quad$ SPA-U based system

- Legacy system compatibility

- Power management for USB based components

Power Management (PM)

- $1500 \mathrm{~W}$ system

- Triple junction deployed solar array

- Lithium-ion batteries

- Peak power tracking

Structure

- Aluminum honeycomb

Propulsion

- No onboard propulsion system

Again using these requirements, components were selected for each subsystem. The resulting mass, power, and volume require-ments for the high capability system are summarized on Table 2.

Table 2: High Capability Bus

\begin{tabular}{|l|c|c|c|}
\hline Subsystem & $\begin{array}{c}\text { Mass } \\
{[\mathrm{kg}]}\end{array}$ & $\begin{array}{c}\text { Power } \\
{[\mathrm{W}]}\end{array}$ & $\begin{array}{c}\text { Size } \\
{[\mathrm{cm}]}\end{array}$ \\
\hline ADC & 23.3 & 49.5 & $35 \times 35 \times 22$ \\
\hline TTC & 10.6 & 64.4 & $25 \times 25 \times 15$ \\
\hline NG & 0.0 & 0.8 & $7.0 \times 4.5 \times 1.0$ \\
\hline CDH & 15.2 & 50 & $34 \times 25 \times 20$ \\
\hline PM & 54.6 & 253 & $72 \times 23 \times 21$ \\
\hline Structure & 38.6 & n/a & $52 \times 40.5 \times 71$ \\
\hline Propulsion & 0 & 0 & $0 \times 0 \times 0$ \\
\hline \multirow{2}{*}{} & $\mathbf{1 4 2 . 3}$ & $\mathbf{4 1 7 . 7}$ & $\mathbf{5 2 \times 4 0 . 5 \times 7 1}$ \\
\cline { 2 - 4 } & \multicolumn{3}{|r}{}
\end{tabular}

The total power loads summarized on Tables 1 and 2 represent the maximum heat load for the system. Because the majority of the subsystems consist of components that are subject to electrical losses rather than mechanical devices, nearly $90 \%$ of the power generated by the satellite must be radiated to space by the TCS. Most thermal engineers for small satellites assume $100 \%$ power dissipation for the hot case to provide additional margin to the design. As for the cold case, the satellite never completely shuts down so the internal heat load is always greater than $0 \mathrm{~W}$. The actual value is dependent on the satellite and the mission, but in general the lowest value that can be expected is $50 \mathrm{~W}$.

Navigation and Guidance (NG) 


\section{PAYLOADS}

The fundamental goal of ORS is to provide the advantages that strategic commanders depend on to the commanders in the field. The primary missions of interest are Intelligence, Surveillance, Reconnaissance (ISR); communications; navigation supplementation; and Blue Force Tracking (BFT). Each of these missions dictates different payloads that must be flown, and each payload levies its own set of requirements on the bus and the thermal control system.

Unfortunately, the detailed requirements for each payload do not exist making it difficult to integrate the payload into the thermal design of the system. For the other subsystems, this does not necessarily pose a significant problem because their interface can be defined by a set of standards. The best examples are the $\mathrm{CDH}$ system, the power system, and the satellite structure. For the thermal control system this can pose a significant challenge if the TCS for the bus must also provide proper temperature control for the payload. Specific requirements would have to be detailed for each individual payload.

An alternative solution is to treat the payload to bus interface in an analogous manner to that of the $\mathrm{CDH}$ and power systems. Instead of forcing the TCS to control the temperature of the payload, a set of interface standards will be developed that dictates the maximum allowable power dissipation, the minimum allowable power dissipation, and the nominal interface temperature limit. Essentially, the payload would be treated as an external load on bus. This concept provides adequate definition for the development of the TCS while allowing for payload flexibility.

Under this approach, the design and engineering of the payload is essentially separated from the bus. The bus would provide a specific range of baseline capabilities to meet the needs of most missions and payloads. The goal is an $80 \%$ design solution. Any additional capability required by the payload would have to be provided by the payload itself. Integration of the bus and payload would occur through standard interfaces.

\section{INTERFACES}

As discussed previously, thermal interfaces will play an important role for responsive satellites. In order to be successful, interface standards will have to be developed between components and mounting surfaces, between subsystems and the bus structure and between the bus and the payload. Standardized interfaces will facilitate the rapid design, analysis, fabrication, and test of the satellite bus and payload.

One advantage to the modular system approach is that it simplifies integration by reducing the number of interfaces. By separating at the subsystem level, the thermal design is separated into two parts at a natural break point, which are the overall bus design and the component specific design. Rather than having to specify interface standards for every type of component, standards would only have to be created for the subsystem/bus interface. By separating at that location, the subsystem supplier would be responsible for developing the thermal design of the components inside the enclosure; whereas, the system integrator would be responsible for developing the overall thermal control of the bus. The interface between the bus and the subsystems would be dictated by a thermal design standard that both parties would have to follow.

\section{EXTERNAL ENVIRONMENT}

For most spacecraft, the thermal environment and the external heat loads are determined from the specific orbit for the mission, the orientation of the spacecraft, the surface properties, and the size of the system. From these, the absolute worst hot and cold case conditions are determined. Unfortunately, none of these parameters are clearly defined for ORS missions. Since specific orbits are largely unknown for ORS, the TCS must be adaptable to all low earth orbits. The only constraining assumption that can be made is that the orbit regime is limited to low Earth orbits. For simplicity, only circular orbits were evaluated.

Using these constraints, the worst hot case condition is shown on Fig. 2 and is defined below ${ }^{5,6}$.

- Orbit beta angle is $90^{\circ}$.

- Eclipse duration is zero.

- The panel with the largest surface area is always nadir facing.

- The panel with the second largest surface area always faces the sun.

- Solar flux is $1414 \mathrm{~W} / \mathrm{m}^{2}$.

- $\quad$ Earth IR is $275 \mathrm{~W} / \mathrm{m}^{2}$.

- Albedo coefficient is 0.57 .

- The side reserved for the payload faces space. That side does not radiate heat to space. 


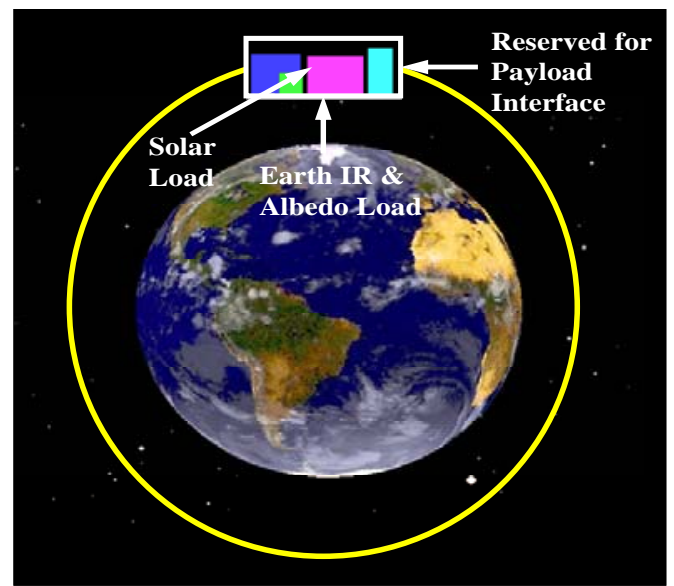

Figure 2: Worst Hot Case Orientation

The worst cold case condition is shown on Fig. 3 and is defined below ${ }^{5,6}$.

- Orbit beta angle is $0^{\circ}$.

- Eclipse duration is $43 \%$.

- The panel with the smallest surface area is always anti-nadir.

- Solar flux is $1322 \mathrm{~W} / \mathrm{m}^{2}$.

- The side reserved for the payload is nadir pointing, so there is not an Earth IR or Albedo heat load.

It is important to note that these are theoretical worst case conditions. For example, it is unlikely that both the Albedo and Earth IR maximum heat loads will occur at the same time. The Albedo heat load increases with orbit inclination; whereas, the Earth IR heat load increases with decreasing orbit inclination. The theoretical worst case scenarios were chosen to provide confidence in the design and to add a significant amount of margin for most orbits.

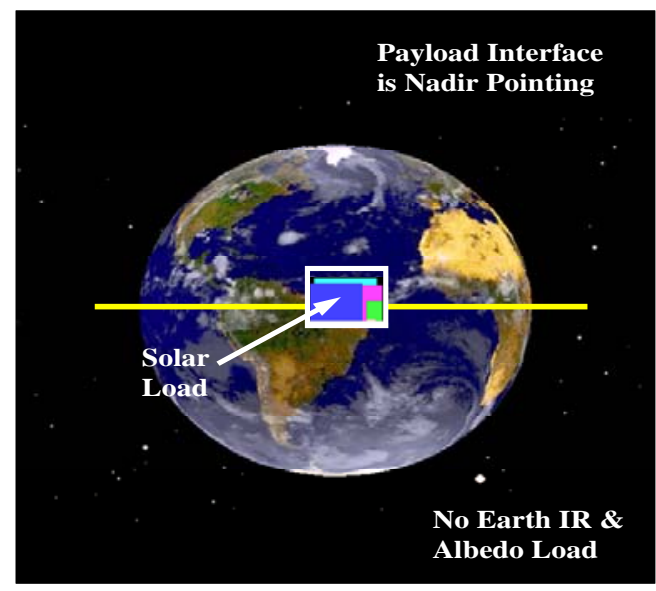

Figure 3: Worst Cold Case Orientation
Finally, it is important to address the transients of the low Earth orbit (LEO) environment. Because of the low altitudes and short orbital periods, the LEO environment is dynamic and creates special difficulties for the thermal engineer. A LEO spacecraft only sees a small portion of the Earth. As it orbits, it is exposed to rapidly changing environmental conditions as it passes over various geographical features and local time zones, which significantly affect Earth IR and Albedo heat loads. In addition, eclipse times can vary from nearly half of the orbital period to zero. As a result, the thermal capacitance of the system is important, especially for lightweight components.

The focus of this effort is on the core bus structure and not external component such as solar arrays or antennas. For this reason, orbit averaged values were used because of the large thermal capacitance associated with the bus. To validate this assumption, a first order transient analysis was conducted.

The transient behavior for a radiation-conduction system is determined using the following equation

$t_{r-c}=\frac{m c}{3 \varepsilon \sigma A_{\text {rad }}}\left(\frac{1}{T^{3}}-\frac{1}{T_{i}^{3}}\right)$

where $\mathrm{m}$ is the mass of the system $[\mathrm{kg}], \mathrm{c}$ is the specific heat $[\mathrm{J} / \mathrm{kg}-\mathrm{K}]$, and $\mathrm{T}_{\mathrm{i}}$ is the initial temperature ${ }^{7}$. The equation assumes the temperature of the surroundings is $0 \mathrm{~K}$, which is valid for a first order approximation. Using $875 \mathrm{~J} / \mathrm{kg}-\mathrm{K}$ for the specific heat of aluminum and the modified density of the bus (total mass divided by total volume), the time for the temperature of the low capability bus to change from $303 \mathrm{~K}$ to $273 \mathrm{~K}$ is 81 minutes. For the high capability bus, the change occurs in 120 minutes. For a satellite in LEO at an altitude of $200 \mathrm{~km}$ and a $\beta$ angle of $0^{\circ}$, the eclipse time is only 36 minutes. Therefore, orbit averaged values for the external heat loads are acceptable.

\section{TEMPERATURE LIMITS}

Before evaluation of the system architecture can be initiated, there is one remaining topic that must be addressed. The fundamental purpose of the TCS is to maintain components within their acceptable operating and survival temperature limits. These limits are wide ranging and component dependent. To ensure that all of the components are within their operating temperature limits the components with the tightest temperature range were used to define the temperature requirements of the system. For this case, they were the 
momentum wheel and Lithium ion batteries, which have an operating temperature range from 263 to $313 \mathrm{~K}$.

Because of uncertainties within the TCS, it is common practice to add margin to the temperature constraints. A study of a number of military programs concluded that an $11 \mathrm{~K}$ margin was required to provide $95 \%$ confidence that flight temperatures would be within limits for a model correlated to thermal balance test data $^{8}$. For uncorrelated models, the uncertainty jumps to $17 \mathrm{~K}$. An informal survey of NASA and commercial satellite programs showed that $5 \mathrm{~K}$ was the most common margin used ${ }^{6}$. Because the external and internal heat load values chosen for the hot and cold case analyses are already conservative, a $10 \mathrm{~K}$ margin will be used even though the model will be uncorrelated.

\section{ENERGY BALANCE}

Essentially, the primary task of the thermal engineer is to balance the thermal energy of the satellite to ensure all of the internal components remain within their acceptable temperature limits during the worst hot and cold cases. External and internal heat generation must be properly balanced with the excess heat radiated to space. A simple energy balance analysis between the satellite and the space environment can be used to determine whether or not the satellite has enough surface area to maintain its temperature within acceptable limits for the hot case. In addition, it can be used to size survival heater power to maintain the temperature within acceptable limits for the cold case.

The actual temperature of space is $4 \mathrm{~K}$; however, as a first order approximation the temperature of space can be assumed to be $0 \mathrm{~K}$. Substituting in expressions for the heat loads, the energy balance equation is ${ }^{9}$ :

$$
\begin{aligned}
& \varepsilon \sigma A_{\text {rad }} T_{S}^{4}=\varepsilon A_{S} F_{S, e} I_{E I R}+\alpha A_{\perp} I_{\text {sun }}+ \\
& \alpha a A_{S} F_{S, s e} I_{\text {sun }}+Q_{\text {Internal }}
\end{aligned}
$$

where $\varepsilon$ is the emissivity of the spacecraft, $\sigma$ is the Stefan-Boltzmann constant $\left[\mathrm{W} / \mathrm{m}^{2}-\mathrm{K}^{4}\right], A_{\text {rad }}$ is the radiator surface area $[\mathrm{m}], T_{s}$ is the average temperature of the spacecraft $[K], A_{s}$ is the surface area $[\mathrm{m}], \mathrm{F}_{\mathrm{s}, \mathrm{e}}$ is the view factor between the spacecraft and the Earth, $I_{E I R}$ is the intensity of the Earth IR, $\alpha$ is the surface solar absorptivity, $A_{\perp}$ is the area perpendicular to the sun $[\mathrm{m}]$, and $\mathrm{I}_{\text {sun }}$ is the solar heat flux $\left[\mathrm{W} / \mathrm{m}^{2}\right], a$ is the Earth albedo coefficient, $F_{\mathrm{s}, \mathrm{se}}$ is the view factor between the spacecraft and the sunlit Earth, and $\mathrm{Q}_{\text {Internal }}$ is the internal heat generation [W]. This equation provides a first order approximation of the radiator area need for the hot case and the heater power needed for the cold case.

\section{Energy Balance for the Low Capability Bus}

By rearranging Eq. 2 and solving for $A_{\text {rad }}$, the radiator surface area required to keep the satellite below the maximum operating temperature during the hot case condition can be calculated. The cold case temperature is also determined using Eq. 2 by solving for $T_{s}$. If the temperature exceeds the lower temperature limit, survival heaters must be used to provide additional heat. Using Eq. 2 to determine the radiator area and the survival heater power for the satellite provides a first order approximation to size the TCS. It also provides a tool to quickly eliminate thermal control schemes and hardware that will not be applicable to the problem.

For the first order approximation of the energy balance, the internal heat load for the hot case, which was summarized on Table 1, is $147.0 \mathrm{~W}$. As for the cold case, the internal heat load was assumed to be $50 \mathrm{~W}$. Next, it was assumed that the surface was painted white, and only five surfaces were available for radiation to space. The remaining surface was reserved as the interface surface for the payload. An emissivity of 0.88 and an absorptivity of 0.22 were used for white paint. The inputs into the energy balance equation are summarized below.

Table 3: Summary of the Inputs for the Energy Balance Equation for the LCB

\begin{tabular}{|l|c|c|}
\cline { 2 - 3 } \multicolumn{1}{c|}{} & Hot Case & Cold Case \\
\hline Eclipse Percent & 0 & 0.43 \\
\hline Solar Constant $\left[\mathrm{W} / \mathrm{m}^{2}\right]$ & 1414 & 1322 \\
\hline Albedo Coefficient & 0.57 & 0.18 \\
\hline Earth IR $\left[\mathrm{W} / \mathrm{m}^{2}\right]$ & 275 & 218 \\
\hline Internal Heat $[\mathrm{W}]$ & 147 & 50 \\
\hline Temperature Limit $[\mathrm{K}]$ & 303 & 273 \\
\hline Area $\perp$ to Sun $\left[\mathrm{m}^{2}\right]$ & 0.192 & 0.109 \\
\hline Area $\perp$ to Earth $\left[\mathrm{m}^{2}\right]$ & 0.288 & 0 \\
\hline
\end{tabular}

Using the energy balance equation and the parameters above, the radiator area required to keep the bus below $303 \mathrm{~K}$ was $0.76 \mathrm{~m}^{2}$ and the resulting cold case temperature was $204.6 \mathrm{~K}$. The total available radiator area of the bus was $1.07 \mathrm{~m}^{2}$. If the surface area was increased to the total available radiator area, the hot case temperature was reduced to $278.3 \mathrm{~K}$, and the cold case temperature was reduced to $187.9 \mathrm{~K}$. To increase 
the cold case temperature to acceptable levels, a survival heater power of $240 \mathrm{~W}$ would be required. A passive thermal control system incorporating survival heaters would satisfy the thermal requirements. However, an active system might be needed because of the large survival heater power requirement.

\section{Energy Balance for the High Capability Bus}

For the high capability bus, the internal heat loads for the hot case and cold case were $417.7 \mathrm{~W}$ and $50 \mathrm{~W}$, respectively. Again, it was assumed that only five sides of the satellite were available for radiation to space, the surface finish was white paint, and the temperature limits remain unchanged. All of the input values are summarized on Table 4. Following the same process as before, the radiator area required to keep the bus below $303 \mathrm{~K}$ was $1.59 \mathrm{~m}^{2}$; however, the available radiator area was only $1.52 \mathrm{~m}^{2}$. The result was a hot case temperature of $306.5 \mathrm{~K}$. The cold case temperature was $183.0 \mathrm{~K}$, and the survival heat power was $360 \mathrm{~W}$. Because the system was already above the maximum temperature limit, supplemental radiator area was required.

Table 4: Summary of the Inputs for the Energy Balance Equation for the HCB

\begin{tabular}{|l|c|c|}
\cline { 2 - 3 } \multicolumn{1}{c|}{} & Hot Case & Cold Case \\
\hline Eclipse Percent & 0 & 0.43 \\
\hline Solar Constant $\left[\mathrm{W} / \mathrm{m}^{2}\right]$ & 1414 & 1322 \\
\hline Albedo Coefficient & 0.57 & 0.18 \\
\hline Earth $\mathrm{IR}\left[\mathrm{W} / \mathrm{m}^{2}\right]$ & 275 & 218 \\
\hline Internal Heat $[\mathrm{W}]$ & 417.7 & 50 \\
\hline Temperature Limit $[\mathrm{K}]$ & 303 & 273 \\
\hline Area $\perp$ to Sun $\left[\mathrm{m}^{2}\right]$ & 0.287 & 0.211 \\
\hline Area $\perp$ to Earth $\left[\mathrm{m}^{2}\right]$ & 0.392 & 0 \\
\hline
\end{tabular}

\section{THERMAL CONTROL SYSTEM ARCHITECTURE}

Once the initial energy balance for the system was completed, the focus of the effort turned to the thermal control system architecture. Since the focus of ORS is to deploy a spacecraft within six days of call-up, the primary design drivers of the system are modularity and ease of integration. To enhance storability, transport, and integra-tion, the subsystems were housed in separate enclosures. The actual layouts for the LCB and $\mathrm{HCB}$ are shown on the figure below.
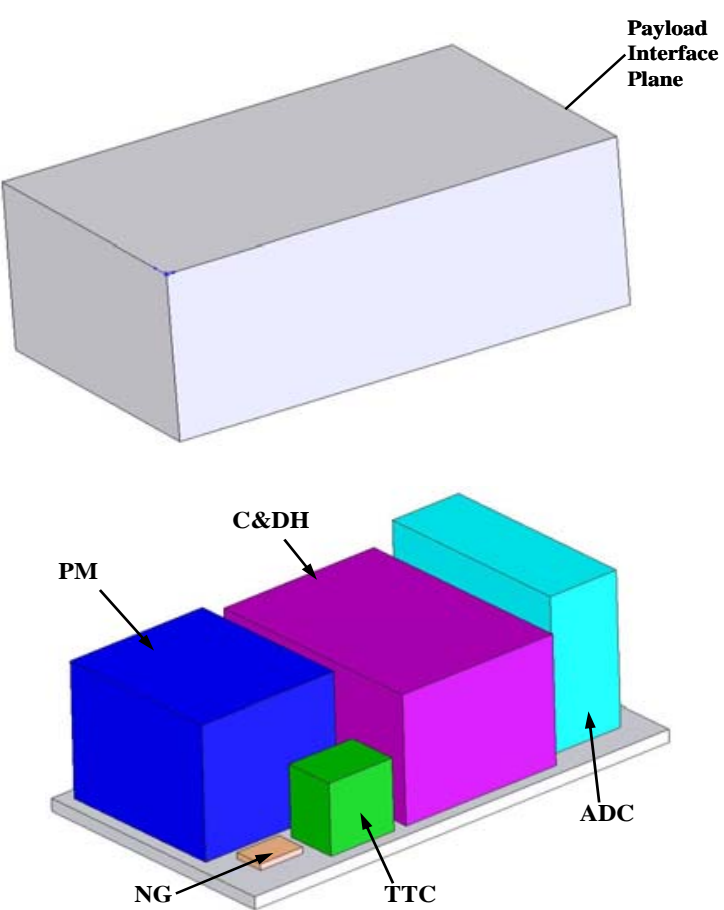

Figure 4: Layout of the LCB
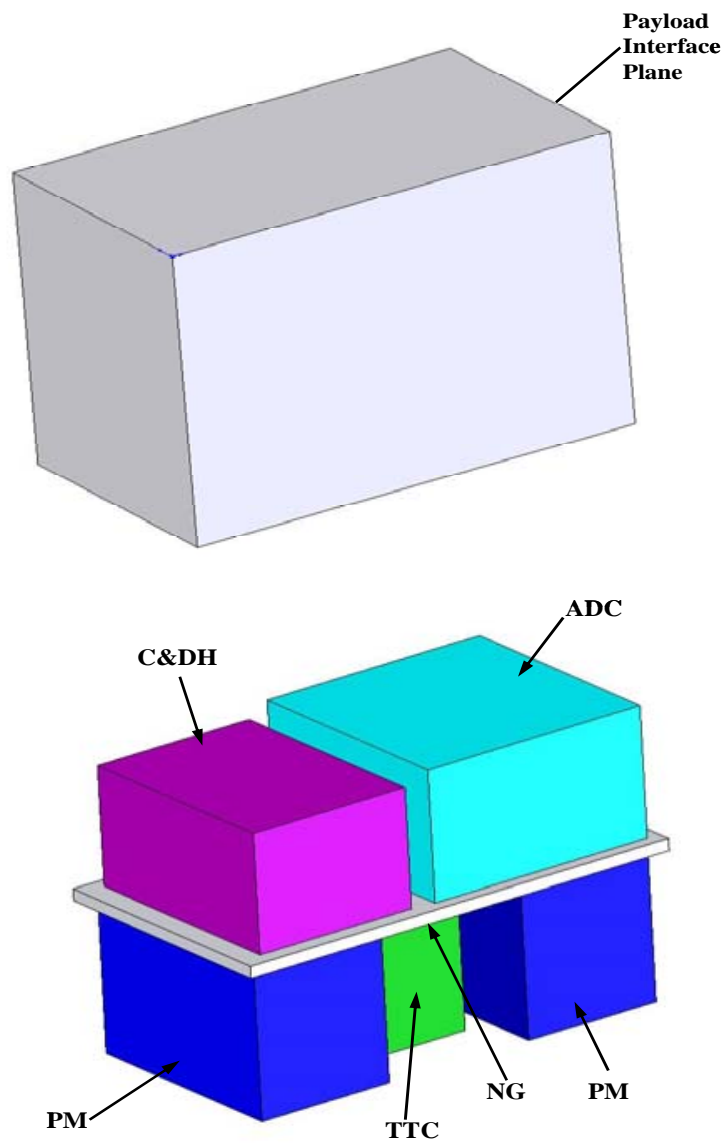

Figure 5: Layout of the HCB 
Because the subsystems were housed in separate enclosures, the design of the TCS was split into two parts. The first part, which focused of the design of the overall bus TCS, emphasized the conduction of heat from the subsystems through the bus structure to the exterior of the satellite where it can be radiated to space. Initially, the subsystems were modeled as simple aluminum enclosures with uniform heat loads. After the heat conduction path through the bus was designed, the focus turned to the subsystems, which was the focus of the second part of the design. Finally, the bus model and the subsystem models were integrated, and the final design was analyzed.

Initially three basic TCS architectures were investigated. The first was an isothermal architecture where panels with high thermal conductivity inserts were used to spread heat across the entire satellite. The second was a thermally isolative approach where each subsystem was isolated from one another and mounted to a dedicated radiator area. The final architecture consisted of a variable heat transfer rate approach, which can be achieved with either a passive heat switch or an active system.

\section{Isothermal Architecture}

To achieve isothermal conditions, the design incorporated a honeycomb electronics shelf with an Annealed Pyrolytic Graphite (APG) core to improve the lateral conductivity of the panel. The design was based on k-Technologies' k-Core concept, which uses encapsulated APG to spread heat across the panel ${ }^{10}$. The lateral conductivity of APG is on the order of 1700 $\mathrm{W} / \mathrm{m}-\mathrm{K}$. A schematic of the k-Core concept is shown in below.

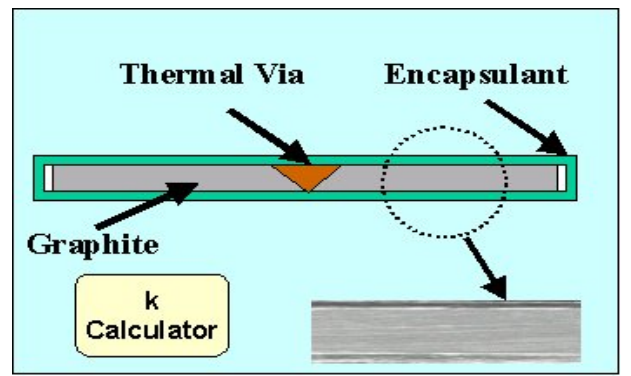

Figure 6: k-Technologies Patented k-Core Material System $^{10}$

The bus structure and the subsystem en-closures were modeled in Thermal Desktop (TD). The subsystems were modeled as Al-2024 enclosures with wall thicknesses of $1.5875 \mathrm{~mm}$ and a thermal conductivity of $185 \mathrm{~W} / \mathrm{m}-\mathrm{K}$. The edges between the different sides of the enclosures were assumed to be in perfect contact, which is the equivalent of a continuous material around the corners. The conductivity of the interface between each of the subsystem enclosures and the shelf was controlled using surface contact conductors. Conservatively, a joint conductivity of $110 \mathrm{~W} / \mathrm{m}-\mathrm{K}$ was assumed for bare aluminum interfaces ${ }^{6}$. As for the electronics shelf, it was modeled as an aluminum honeycomb panel with a $1 \mathrm{~mm}$ APG core encapsulated in the face sheets. As a result, the face sheets for the electronics shelf were 2.6 times thicker than the face sheets used for the other panels. The interface conductivity between the face sheets and the core was controlled using surface contact conductors. The other panels were modeled as two face sheets with a contactor to control the conductivity of the honeycomb core. Initially, a honeycomb core transverse conductance of $250 \mathrm{~W} / \mathrm{m}^{2}-\mathrm{K}$ was used. The panels were also assumed to be in perfect contact with one another.

As for the boundary conditions, the internal heat loads for each subsystem were evenly distributed over all six surfaces of the enclosure. The external loads were applied using surface heat loads. The solar loads for the hot and cold cases were $312 \mathrm{~W} / \mathrm{m}^{2}$ and $166 \mathrm{~W} / \mathrm{m}^{2}$, respectively. The combined Earth IR and Albedo load for the hot case was $414 \mathrm{~W} / \mathrm{m}^{2}$. White paint with an $\varepsilon$ of 0.88 and an $\alpha$ of 0.22 was used for the exterior of the satellite. RadCAD was used to calculate the radiation exchange factors with space. Radiation within the bus was included in the calculations. The interior surfaces were painted black to enhance radiative heat transfer.

Because of the low internal heat density of the LCB, the design was fairly simple. The ADC, TTC, and NG subsystems could be maintained within proper temperatures with a bare aluminum-aluminum interface between the enclosure and the electronics shelf. As for the CDH and PM subsystems, an RTV insert was required to increase the contact conduction at the interface. The design of the HCB was more complicated in that three deployable radiators had to be added to achieve proper cooling. The radiator locations are shown on Figure 7 and are $0.35 \mathrm{~m}$ by $0.405 \mathrm{~m}$. An adequate thermal design could be achieved for the HCB if the solar and the combined Earth IR and Albedo loads were eliminated through the use of Multi-Layer Insulation (MLI). The use of MLI was not considered practical for ORS operations because of its complicated fabrication process, high touch labor, and fragility. Also, since orbits, missions, orientations, and components are unknown, its pre-application to the structural panels is impractical. 


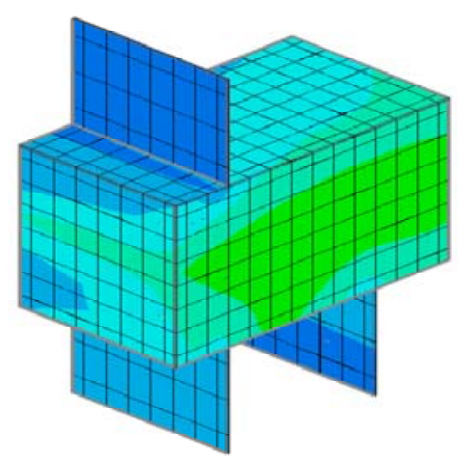

Figure 7: Deployable Radiator Locations

As for the cold case, the temperatures of all of the components were well below the minimum temperature limit without supple-mental survival heater power. To maintain the bus within the baseline temperature limit, an additional heater power of $150 \mathrm{~W}$ was needed for the LCB, which was higher than the total power consumption for the hot case. The HCB required 165 $\mathrm{W}$ of survival heater power.

The high survival heater power requirements are a result of the drastic change in the external load between the hot and cold cases. The same problem was reported by Barton, where survival heater power was $63 \%$ higher than the component operating at full load ${ }^{11}$. As for the worst hot and cold case conditions defined here, it is important to note that it is impossible for both cases to exist for the same orbit. For a more realistic analysis, the worst hot and cold cases were separated by orbit and are outlined below. For each different orbit, the surface properties were tailored and then the heaters were sized.

1a. Worst Case for Hot Orbit: Same as before; results are unchanged

1b. Cold Case for Hot Orbit: Beta angle of $90^{\circ}$, minimum power output, and an orientation with the payload facing the Earth and the smallest adjacent side receiving the solar load

2a. Hot Case for Cold Orbit: Beta angle of $0^{\circ}$, maximum power, and an orientation with largest panels exposed to the solar, Earth IR, and Albedo loads

2b. Worst Case for Cold Orbit: Same as before

For case number one for the LCB, the satellite exterior was painted white, and the survival heater power required was reduced to $30 \mathrm{~W}$. For case number two, the exterior was painted green, which increased the solar absorptivity to 0.57 . The emissivity was unchanged. The survival heater power needed to maintain the minimum temperature was reduced to 40
W. As for the $\mathrm{HCB}$, the survival power requirements were reduced to 115 and $90 \mathrm{~W}$, respectively.

\section{Thermally Isolative Architecture}

To achieve the thermally isolative design, each subsystem was mounted to a different panel. Each panel was then isolated from others with a felt insert at the interface. In this design, the location of the subsystems and the orientation of the satellite play an important role in the design of the TCS, this makes this architecture more difficult to implement. However, since the subsystem properties will be known ahead of time, the majority of the engineering can be performed ahead of time and a fairly simple design analysis and optimization software tool can be completed once the mission is known.

The results for the thermal isolative architecture were similar to the results for the isothermal architecture in that a large survival heater power level was needed to maintain cold case temperatures. Because the system and component location could be optimized somewhat with this architecture, the heater power level was $25 \%$ less. However, this value is still significant compared to the overall power of the buses. This architecture provided an improved thermal performance but will be more difficult to implement under ORS operations.

\section{Variable Heat Transfer Architecture}

This type of architecture can be achieved either with a passive thermal switch, an active convection based system, or a variable emissivity radiator. The key is to change the heat transfer rate between the hot and cold cases. The ideal method would be to implement passive conduction based thermal switches. However, thermal switches have not achieved the reliability necessary for space missions. Instead of basing the analysis on a single technology, a more general analysis was conducted to determine the switching requirements for such architectures.

In the variable heat transfer system architecture, the critical design parameter is the heat transfer from the subsystem through the base plate during the cold case in order to maximize the temperature rise across the system, which minimizes the survival heater power requirement. The temperature rise through the interface between the base plate and the enclosure is calculated with the following equation. 


$$
T_{H}-T_{C}=\Delta T=\frac{Q}{A K_{J}}
$$

where $T_{H}$ is the temperature on the hot side of the joint $[\mathrm{K}], \mathrm{T}_{\mathrm{C}}$ is the temperature on the cold side of the joint $[\mathrm{K}], \mathrm{Q}$ is the heat load [W], $\mathrm{A}$ is the contact area $\left[\mathrm{m}^{2}\right]$, and $\mathrm{K}_{\mathrm{J}}$ is the joint conductivity $\left[\mathrm{W} / \mathrm{m}^{2}-\mathrm{K}\right]$. Since the two interfaces and the interstitial material are in series, their thermal resistances are added. This is analogous to electrical resistances and the same rules apply. Figure 8 provides a schematic for clarity.

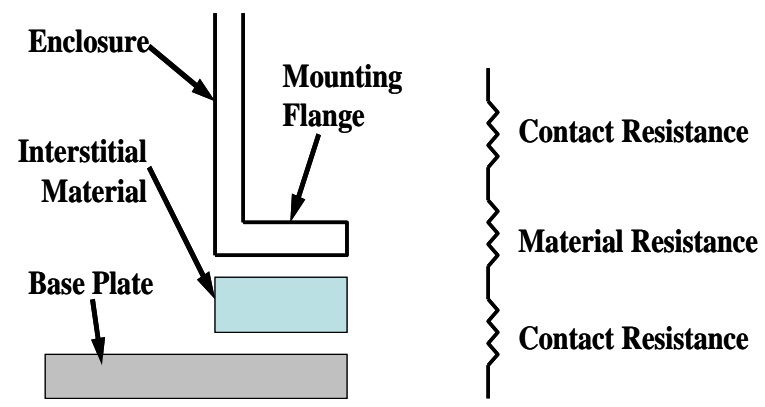

Figure 8: Schematic of the Thermal Joint between the Enclosure and the Base Plate

To be consistent with the electrical resistance analogy, Eq. 3 is modified to the following form:

$$
\Delta T=Q R
$$

where $\mathrm{R}$ is the thermal resistance $[\mathrm{K} / \mathrm{W}]$. The total resistance for the joint is:

$$
R_{\text {Tot }}=\frac{1}{A}\left(\frac{2}{K_{\mathrm{int}}}+\frac{L}{K}\right)
$$

The joint conductivity, $\mathrm{K}_{\mathrm{J}}$, is the inverse of the total joint resistance divided by the contact area.

Since the temperature on the hot side of the interface is dependent on the system parameters i.e. the contact area, the internal power dissipation, and the cold side temperature, it is difficult to identify a single joint conductivity that would meet the thermal needs for all potential components and subsystems. A very small joint conductivity on the order of $1 \mathrm{~W} / \mathrm{m}^{2}-\mathrm{K}$ would probably meet the needs of the majority, but it might not be possible to design a thermal joint with that small of a thermal conductivity. To better gauge the need, the LCB and HCB designs were evaluated. For the LCB, the cold case temperature from the energy balance was $187.9 \mathrm{~K}$. For the HCB, it was $183.0 \mathrm{~K}$. Using the cold case power consumptions and the contact areas for each enclosure, based on the thermal joint above, the joint thermal conductivity required to keep the subsystem temperatures above the lower temperature limit was calculated. The results are presented on Table 5.

Table 5: Joint Conductivity Required to Meet the Minimum Temperature Limit

\begin{tabular}{|c|c|c|c|c|}
\hline System & $\begin{array}{c}\text { Heat } \\
\text { Load } \\
{[\mathrm{W}]}\end{array}$ & $\begin{array}{c}\text { Surface } \\
\text { Area } \\
{\left[\mathrm{m}^{2}\right]}\end{array}$ & $\begin{array}{c}\text { Power } \\
\text { Density } \\
{\left[\mathrm{W} / \mathrm{m}^{2}\right]}\end{array}$ & $\begin{array}{c}\mathrm{K}_{\mathrm{J}} \\
{\left[\mathrm{W} / \mathrm{m}^{2}-\mathrm{K}\right]}\end{array}$ \\
\hline LCB & \multicolumn{5}{|c|}{} \\
\hline ADC & 18.5 & 0.0168 & 1101.19 & 12.80 \\
\hline CDH & 13.0 & 0.0236 & 550.85 & 6.41 \\
\hline PM & 16.2 & 0.0184 & 880.43 & 10.24 \\
\hline TTC & 7.4 & 0.0067 & 1101.19 & 12.80 \\
\hline HCB & \multicolumn{5}{|c|}{} \\
\hline ADC & 18.5 & 0.0228 & 811.40 & 9.43 \\
\hline CDH & 13 & 0.0236 & 550.85 & 6.41 \\
\hline PM & 41.2 & 0.0372 & 1107.53 & 12.88 \\
\hline TTC & 7.4 & 0.016 & 462.50 & 5.38 \\
\hline
\end{tabular}

To meet the needs of all of the subsystems on the table, a joint conductivity of $5 \mathrm{~W} / \mathrm{m}^{2} \mathrm{~K}$ is required; however, this does not take into account the temperature rise from the enclosure to the component and a joint conductivity on the order $10 \mathrm{~W} / \mathrm{m}^{2}-\mathrm{K}$ will probably be acceptable. The design or description of such a joint is beyond the scope of this effort.

For architectures based on thermal switches, the performance of the system is based on the conductance ratio of the system. If the conductance ratio is high enough, then the need for survival heaters is virtually eliminated. The result would be a very robust system. Conductance ratios on the order of $20: 1$ to $70: 1$ are needed for a robust operational system.

There is one disadvantage to this system architecture. The first is that the switching component typically adds an additional thermal interface to the system. For radiator panels that are already operating at their limit, adding the additional interface will cause the components to exceed their operating temperatures during the hot case. As a result, radiator area has to be oversized to ensure proper operation, which will add some mass to the system. However, the advantage of a modular, robust system outweighs the disadvantages when a short turn-around-time becomes more important than mass. 


\section{TESTING}

In addition to reducing the time needed for design and modeling of the satellite and the TCS, the time need for testing must also be reduced. A modular ORS system will have limited utility if the design, modeling, and fabrication of the system can be completed in a matter of days or weeks but it takes another six months to complete the testing and validation of the system.

Testing is currently, and will always be, a critical component of TCS development. It is used for model validation, design verification, component screening, and craftsmanship quality assurance. The latter one is especially important for multi-layer insulation and the thermal joints within the satellite. For ORS to succeed, testing methodologies and techniques will also require a significant paradigm shift. Rapid prototype testing and built-in test capabilities will be required for all temperature critical components, and sensors will need to be integrated into both components and the bus structure.

\section{CONCLUSIONS}

This effort was an initial investigation into the issues and implications that the TCS presents to ORS. It is not meant to be all inclusive but rather a starting point for further analysis and design. To that extent, preliminary thermal requirements and inputs into the design process were identified and evaluated. From there, three different system architectures were evaluated. Of the three, the variable heat transfer architecture was best suited for ORS because of the wide range of components, missions, and orbits envisioned for ORS. This architecture provided the most robust solution. However, with advances in specific technologies, the other two architectures would also be suitable for ORS missions.

As for a one-size-fits-most system, a design solution is possible based on the variable heat transfer architecture. However, its success is dependent on developing either passive thermal switches or lightweight, low power active systems suitable for small satellites.

The thermal control system poses significant challenges to the goals of ORS. Highly optimized systems will not be feasible on the short time scale dictated for tactical satellites. Instead modular, robust, adaptable systems are required. To meet these challenges, two areas of development are critical. The first is system architecture and design tools. The second is the technologies capable of meeting the requirements dictated by the system architectures.

\section{REFERENCES}

1. Rumsfeld, Donald H., Report of the Commission to Assess United States National Security Space Management and Organization, Washington D. C., U.S., Government Printing Office, 2001.

2. Morphopoulus, Thomas, et al., "Plug-and-Play An Enabling Capability for Responsive Space Missions," AIAA $2^{\text {nd }}$ Responsive Space Conference, Paper No. RS2-2004-5002, Los Angeles, CA, 19-22 April 2004.

3. Lyke, Jim, et al., "Space Plug-and-Play Avionics," AIAA $3^{\text {rd }}$ Responsive Space Conference, Paper No. RS3-2005-5001, Los Angeles, CA, 25 - 28 April 2005.

4. Williams, Andrew., Robust Satellite Thermal Control Using Forced Air Convection Thermal Switches for Operationally Responsive Space Missions, Master's Thesis, University of Colorado, Department of Aerospace Engineering Sciences, Boulder, CO, 2005.

5. Wertz, James R., and Wiley J. Larson, Space Mission Analysis and Design, $3^{\text {rd }}$ Ed. Microcosm Press, El Segundo, CA, 1999, pg 433.

6. Gilmore, David G., Spacecraft Thermal Control Handbook Volume I: Fundamental Technologies, $2^{\text {nd }} E d$, The Aerospace Press, El Segundo, CA, 2002, pg 271-272.

7. Incropera, Frank P, and David P. DeWitt, Fundamentals of Heat and Mass Transfer, $4^{\text {th }}$ Ed. John Wiley \& Sons, New York, 1996.

8. USAF, Department of Defense Handbook: Test requirements for Launch, Upper Stage, and Space Vehicles, Vol 1: Baselines, (MIL-HDBK-340A), 01 April 1999.

9. Griffin, Michael D. and James R. French, Space Vehicle Design, AIAA Education Series, Washington D. C., 1991, pg 392-394.

10. k-Technology "k-core" retrieved from http://www.k-technology.com/kcore. html on 21 June 2005.

11. Barton, Mark and Jon Miller, "Modular Thermal Design Concepts: Thermal Design of a Spacecraft on a Module Level for LEO Missions," $19^{\text {th }}$ Annual AIAA/USU Conference on Small Satellites, Paper No. SSC05-I-1, 2005. 